\title{
Retirement and Meaning Attribution: A Study with Active Workers in Brazil
}

\author{
Laura Andrade* (1) \& Cláudio Torres (1) \\ Universidade de Brasília, Brasília, DF, Brasil
}

\begin{abstract}
RESUMO - O objetivo deste estudo foi investigar os significados atribuídos à aposentadoria por trabalhadores ativos. Para isto, foi construída e aplicada a Escala de Significado da Aposentadoria, numa amostra de 275 trabalhadores ativos (53,5\% mulheres) com idades entre 22 e 67 anos. Os resultados revelam o significado ambíguo do fenômeno da aposentadoria: há uma ideia positiva, de liberdade; mas, por outro lado, os trabalhadores relatam angústia e insegurança relacionadas à aposentadoria. Entre as variáveis estudadas, é especialmente relevante o impacto da proximidade da aposentadoria na percepção do fenômeno, que revelou influência sobre três dos quatro fatores identificados. Esta investigação tem implicações para programas e políticas de preparação para a aposentadoria que visam promover a saúde e o bem-estar dos trabalhadores.
\end{abstract} PALAVRAS-CHAVE: aposentadoria, significado, trabalhadores

\section{Aposentadoria e Atribuição de Significado: Um Estudo com Trabalhadores Ativos no Brasil}

\begin{abstract}
The aim of this study was to investigate the meanings attributed to retirement by active workers. For this purpose, the Retirement Meaning Scale was constructed and administered to 275 active workers (53.5\% women) aged 22 to 67 years. The results reveal an ambiguous meaning attribution to the phenomenon: on one hand, there is a positive idea of freedom; on the other hand, the workers report experiences of anguish and insecurity related to retirement. Among the variables investigated, the proximity to retirement was especially relevant to the perception of the phenomenon, which influenced three of the four factors identified. This study has implications for policies and programs that aim to promote healthy experiences in retirement.
\end{abstract}

KEYWORDS: retirement, meaning, workers

Working is an essential part of society organization, being one of the main aspects of human life (Zanelli et al., 2010). In adulthood, working has a central position in individuals' identities (Fouad \& Bynner, 2008), determinant to self-concept (Zanelli et al., 2010), and self-esteem, it reflects individual attitudes, values and political positioning (Magalhães et al., 2004). From a social point of view, working is also recognized as a priority source of social insertion and recognition (Bendassolli \& Gondim, 2014; Bressan et al., 2012; Zanelli, et al., 2010) and it is not unusual that schedules, activities and interpersonal relationships established throughout life are organized around work demands (Zanelli et al., 2010).
For the purposes of this research, working is considered as a parallel concept to employment, a paid activity that involves a working day (Yamamoto, 2015). Bearing in mind that working and employment are central to the organization of communities and to the lives of individuals, and that they are extremely important both as a constituent part of the identity and subjectivity of the worker and as a mediator of workers' life cycles (Bendassolli \& Gondim, 2014), it is expected that one of the main events in the life of an active worker is the retirement process (Bressan et al., 2012; Duarte, \& Melo-Silva, 2009).

In recent years, retirement has become a major theme in career planning (Rodrigues et al., 2014). This stage presents

*E-mail: lauranovaesa@gmail.com

- Submetido: 29/12/2017; Revisado: 19/09/2018; Aceito: 12/11/2018. 
itself as a complex paradox for both scholars and workers themselves, since retirement can have either a positive meaning of freedom, being a desired moment in which the individual can devote himself to personal projects, family, and self-care; or it might have a negative meaning, associated to withdrawing, and marginalization.

From the etymological point of view, the word "retirement" carries within itself the dual meanings of this phenomenon: on the one hand, retirement refers to jubilation - joy and freedom; while, on the other hand, it refers to retreating to the non-working space, often associated with abandonment, inactivity and finitude (Leandro-França, 2016). Since language is an important form of expression of shared meanings and cultural transmission (Triandis, 2002), naming social phenomena with specific words is not a random or naïve action - the words of each language very effectively reflect the meaning that that group attributes to the social objects that surround them. In Brazil, the meaning and more negative experience of the word have been evidenced in numerous studies (Denton \& Spencer, 2009; França et al., 2013; Zanelli, 2012).

Although there has been a significant increase in publications concerning retirement in recent years (Fisher et al., 2016; França et al., 2013; Rodrigues et al., 2014), this is still a new research topic, with several possibilities of study and intervention yet to be explored. In a recent review, Boehs et al. (2017) identified only 42 articles dealing with the theme of retirement from the perspective of psychology in Latin America literature - 24 of which were published after 2016. Thus, it is an incipient but prosperous field for the psychologist's work.

Even from the historical point of view retirement is a very recent fact in Brazil (Oliveira et al., 2009; Shultz \& Wang, 2011). Only in 1974 the Ministry of Social Security and Welfare was instituted in the country, and it defined social security as the guarantee assured to workers who paid their taxes that they will have their income replaced by a social insurance payment when they lose their working capacity due to illness, disability, aging, death, involuntary unemployment, maternity or imprisonment.

In this study, retirement is understood as the phase of life characterized by the decoupling of work activities with rigorous routine commitments that are associated with salary and survival (França et al., 2013; Oliveira, 2018). Therefore, retirement here should not be interpreted as a synonym for the absence of work, but as a differentiated stage of the career. Moreover, retirement comprehends a long process that precedes the end of employment relations by many years, characterized much more as a longitudinal process than as a discrete event in the worker's life (França et al., 2013; Shultz \& Wang, 2011; Zanelli, 2015). Temporally, it is possible to identify at least two distinct moments before retirement (Magalhães et al., 2004): the remote phase when the individual recognizes retirement as a distant event and generally sees it positively; and the approximate phase - when the individual is already able to identify a probable retirement date and raises expectations towards job termination and the social situations that surround it (Oliveira, 2018).

The way this event occurs and the workers' perception about it depends on the demographic, historical, social, economic, and political context in which the worker is inserted (Antunes et al., 2015; França et al., 2013; França et al., 2017; Guerson et al., 2018). On that matter, gender is an important variable for retirement experiences: retirement is expected to have a more negative impact on men (Fernandes \& Garcia, 2010), as women cultivate more intense family bonds that provide greater social support in retirement (Debert, 2013). Similarly, the financial impact of retirement seems to be differentiated between men and women so that there is a negative impact $(-0.16 \%)$ on the value of the insurance received if the worker is a woman (Dias, 2017); such disparity may be related to the way women were inserted in labor market, to the double and triple shifts associated to domestic activities, to the kind of tasks and jobs designated to this gender identity, and to the men's domination of the general market (Barreto \& Heloani, 2018; Yannoulas, 2018).

Overall, retirement is an ambivalent phenomenon: it is a worker's conquest, and, at the same time, represents the marginalization of the inactive worker by the productive society (Guerson et al., 2018; Leandro-França et al., 2014; Oliveira et al., 2009). For some workers, retirement is seen as a phase of relaxation and enjoyment earned after many years of work (Shibata, 2006; Macêdo et al., 2017). This phase involves the possibility of carrying out abandoned projects, the dedication to voluntary or family activities and, to a certain extent, the expectation of positive experiences (Cavalcante \& Minayo, 2012; Macêdo et al., 2017). For another portion of workers, it has a connotation of unproductivity and personal devaluation (Shibata, 2006): the end of the formal work life indicates potential suffering, insecurity, fear, and anguish (Cavalcante \& Minayo, 2012). In that way, retirement can mean loss of status, friends and social reference, and changes in values, norms and routine. It is often a phase associated to the loss of meaning in life, especially regarding social interactions.

In recent years, studies have highlighted the uncertainty and anxiety surrounding the retirement phenomenon (Denton \& Spencer, 2009; Duarte \& Melo-Silva, 2009; França et al., 2013; Zanelli, 2012), the impact of retirement on the dynamics of workers (Antunes et al., 2015), retirementrelated identity metamorphoses (Fernandes et al., 2016), among other related topics.

Some studies were also dedicated to verifying the meaning of retirement. Felix and Catão (2013) verified the coexistence of positive and negative meanings of retirement among road police officers, oscillating between perspectives of exclusion and illness, and freedom. In a similar proposal, Machado and Lucas (2017) found the same expectation of 
freedom linked to retirement and changes in the social and relational dynamics of workers among teachers. However, the studies did not use a specific instrument to measure the meaning of retirement, which leads to the following question: would it be possible to construct a measure to identify the construction of this meaning?

The process of meaning attribution is one of the most important processes of human social activity (Osgood et al., 1957), since the meaning that individuals ascribe to the objects and social situations surrounding them, and the changes in that meaning usually determine how these individuals behave in society. Meaning attribution is a mediating cognitive state between external stimulus, internal reactions, and the person's representation of reality (Pasquali, 2010); it is an interpretation that the individuals make of the stimuli that surround them (Richins, 1994).

For the retirement phenomenon there are basically two possibilities of meanings - positive meanings and negative meanings (Cavalcante \& Minayo, 2012; Leandro-França et al., 2014; Oliveira, 2018). The positive meanings attributed to retirement generally refer to the notion of retirement as a worker achievement/ reward, freedom and autonomy to manage one's life, the possibility of investing in personal projects and other activities, and the resumption of ties. affective and family.

The negative meaning, on the other hand, refers to the financial and health problems present in this phase of life, the loss of social status, the loss of the group of friends, the feeling of worthlessness and idleness, among other aspects. One consequence of this expectation of negative experiences is anxiety related to the event, a widespread feeling of apprehension regarding uncertainties, unpredictability, and negative consequences of retirement (Duarte \& Melo-Silva, 2009; Fletcher \& Hansson, 1991), due to the stigma of inactivity associated with the phenomenon (Fernandes et al., 2016).

Considering the importance of studying the meaning of retirement as predictors of positive or negative experiences of retirement, we are concerned about understanding the process of attributing meaning to retirement in order to understand people's behavior towards the phenomenon, and thus contribute to the construction of effective retirement preparation policies that might help reduce the incidence of suffering, and increase the quality of life in retirement.

Until 2018, some Brazilian instruments aimed to investigate attitudinal changes in relation to retirement (França, 2008) and their predictors (França, 2009), key factors (França \& Carneiro, 2009) and other scales related to planning for retirement (Leandro-França et al., 2014). In 2017, Rafalski et al. identified, in a cross-cultural survey, the existence of 28 retirement-related attitudes, planning, decision-making, adaptation and satisfaction measures published in Portuguese and English. However, no indigenous instrument has been found that addresses the meaning attributed to retirement

Thus, the aim of the present research was to construct and search for validity evidences of a retirement meaning scale for active workers and to investigate the meanings attributed to retirement by this population. Also, the results of this study hope to provide subsidies for the elaboration of retirement preparation policies that promote workers' health and well-being.

\section{METHOD}

\section{Stage I: Qualitative Research and Scale Construction}

In the first stage of the study, there were 40 participants, 24 women and 16 men, aged between 23 and 54 years $(\mathrm{M}=44.7$ years; $\mathrm{SD}=8.7$ years). The participants had, on average, 22.4 years of work ( $\mathrm{SD}=8.5$ years) and $55 \%$ intended to retire in the next ten years. The participants were presented a virtual questionnaire containing four open-ended questions that sought to embrace retirement meanings, feelings, and attitudes: "What does retirement mean to you?", "What do you feel when you think of retirement?", "What are the positive aspects of retirement?" and "What are the negative aspects of retirement?". The answers obtained were analyzed using a thematic categorical content strategy, as proposed by Bardin (1977).

Each question was analyzed separately generating specific categories and themes of their answers. Were found 15 categories and 35 themes (Table 1). These results were used to support the construction of the Retirement Meaning
Scale (RMS) proposed here. Details of the construction, application and search for evidence of validity of this instrument are described below.

\section{Constructing the Scale}

For this measurement, a horizontal rating scale was chosen - a differential semantic scale on which pairs of opposite sentences are used to cover aspects of the meaning attribution process (Osgood, 1952; Pasquali, 2010) to include more complex reflections and evaluations about retirement as a social and individual phenomenon. Thus, in each item, participants were presented with two opposing statements about retirement (eg, "Retirement is a phase of joy" versus "Retirement is a phase of sadness") between which subjects should indicate, on a six-point scale, the statement better aligned with their personal beliefs.

The results of the previous qualitative research in conjunction with the theoretical perspective already described, served as foundation to the development of the first version of the RMS. This version included 62 items 


\begin{tabular}{|c|c|c|}
\hline Question & Category & Themes \\
\hline \multirow{6}{*}{$\begin{array}{c}\text { What does retirement mean to } \\
\text { you? }\end{array}$} & \multirow{4}{*}{ Finish work life $(n=29)$} & Conquest/Right $(\mathrm{n}=13)$ \\
\hline & & Rest time $(\mathrm{n}=4)$ \\
\hline & & Freedom $(n=8)$ \\
\hline & & Quitting work activities $(\mathrm{n}=4)$ \\
\hline & \multirow{2}{*}{ Start new activities $(\mathrm{n}=22)$} & Make new plans $(\mathrm{n}=15)$ \\
\hline & & Prioritize personal issues $(\mathrm{n}=7)$ \\
\hline \multirow{13}{*}{$\begin{array}{l}\text { What do you feel when you } \\
\text { think about retirement? }\end{array}$} & \multirow[t]{2}{*}{ Others } & Others \\
\hline & & Joy and happiness $(\mathrm{n}=6)$ \\
\hline & \multirow{4}{*}{ Positive feelings $(n=24)$} & Accomplishment $(\mathrm{n}=5)$ \\
\hline & & Freedom $(n=4)$ \\
\hline & & Rest perspective $(\mathrm{n}=9)$ \\
\hline & & Undistinguished feeling of fear and insecurity ( $\mathrm{n}=6$ ) \\
\hline & \multirow{3}{*}{ Insecurity and anxiety $(\mathrm{n}=18)$} & Insecurity towards legal and political aspects $(n=6)$ \\
\hline & & Fear of idleness $(n=4)$ \\
\hline & & Anxiety $(\mathrm{n}=2)$ \\
\hline & \multirow{3}{*}{ Post-career planning $(\mathrm{n}=18)$} & Desire to continue to work $(\mathrm{n}=5)$ \\
\hline & & Invest in personal projects $(\mathrm{n}=9)$ \\
\hline & & Adjustment needs $(\mathrm{n}=4)$ \\
\hline & \multirow{4}{*}{ Investing in other life's areas $(n=24)$} & Others \\
\hline \multirow{6}{*}{$\begin{array}{l}\text { What are the positive aspects of } \\
\text { retirement? }\end{array}$} & & Investing in personal life $(\mathrm{n}=12)$ \\
\hline & & Chance to engage in other activities $(n=7)$ \\
\hline & & Investing in personal relationships $(\mathrm{n}=5)$ \\
\hline & \multirow{2}{*}{ Freedom $(n=20)$} & Free time $(n=9)$ \\
\hline & & Freedom and autonomy to mage one's own life $(n=11)$ \\
\hline & Reward $(n=4)$ & Being rewarded for the years of work $(n=4)$ \\
\hline \multirow{9}{*}{$\begin{array}{c}\text { What are the negative aspects of } \\
\text { retirement? }\end{array}$} & Others & Others \\
\hline & \multirow{3}{*}{ Life Management $(\mathrm{n}=32)$} & Idleness $(n=12)$ \\
\hline & & Financial and insurance losses $(n=16)$ \\
\hline & & Adjustment troubles $(\mathrm{n}=4)$ \\
\hline & \multirow{2}{*}{ Social Aspects $(n=12)$} & Feeling useless $(n=4)$ \\
\hline & & Social isolation $(\mathrm{n}=8)$ \\
\hline & \multirow{2}{*}{ Physical Aspects ( $\mathrm{n}=7$ ) } & Aging $(n=5)$ \\
\hline & & Health issues $(\mathrm{n}=2)$ \\
\hline & Other & Other \\
\hline
\end{tabular}

distributed in seven factors, namely: a) meaning pursuit; $b$ ) social insertion and recognition; c) pleasure and suffering; d) regulation; e) workers' conquest/right; f) retirement preparation; and g) health and self-care.

Semantic Analysis. The first version of the RMS was sent to five evaluators who were aligned with the profile of the population to be studied (active workers in the Brazilian labor market). Each evaluator was instructed to judge the intelligibility of the items, with the possibility of suggesting changes in the sentences presented. All evaluated items were considered adequate and were kept for the second version of the scale.

Experts' Analysis. The second version of the scale was sent to three specialists in social, organizational and work psychology with knowledge either in psychometrics or in work and retirement related themes. The judges were sent a form in which they should evaluate the intelligibility and suitability of the items to the proposed factors. All items to 
which the judges could not agree on were excluded. Thus, 49 items were kept for the third version of the instrument, now divided into six facets (a) social insertion and recognition; b) pleasure and suffering; c) regulation; d) workers' conquest/ right; e) retirement preparation; and f) health and self-care. This was the version used in the subsequent study.

\section{Stage 2:Applying the Retirement Meaning Scale}

\section{Participants}

Took part in this study 275 active workers in the Brazilian market, 53.5\% women, aged between 22 and 67 years $(\mathrm{M}=40.51$ years; $\mathrm{SD}=11.08$ years $)$. Of these workers, 96\% had completed undergrad courses, $69.1 \%$ were employed in the public services. $65.5 \%$ of the sample consisted of White, 26.9\% Bale, 5.1\% Black and 0.4\% Natives. Regarding income, $82.9 \%$ of participants stated that they were above or much above the average salary presented $(\mathrm{R} \$ 1.853,00)$.

\section{Instruments and Data Collection}

The 49-item instrument was available online for data collection. The research was disseminated through e-mail groups and social networks, aiming to reach as many workers as possible. The data obtained were tabulated and analyzed using the statistical analysis softwares IBM SPSS Statistics, version 21, (IBM Corp, 2012) and Factor, version 10.3.01 (Lorenzo-Seva \& Ferrando, 2013).

\section{Data Analysis}

Initially, a parallel analysis was performed with the method of Timmerman and Lorenzo-Seva (2011) to verify the scale factorability. Once the number of factors to be extracted from the instrument was identified, it was performed an Exploratory Factorial Analysis (EFA) conducted in Factor.

The EFA was conducted with Hull's method for factor selection (Timmerman et al., 2011) using Principal Axis Factoring (PAF) extraction with promax rotation. Three criteria were used for the disposal of inefficient items in the questionnaire: (a) exclusion of items with factor loadings lower than 0.30 due to poor representation of the construct (Tabachnick \& Fidell, 2007); (b) exclusion of items that share similar main loads in three or more factors; and (c) exclusion of items that share main loads in two factors with differences smaller than 0.10 between shared loads (Gorsuch, 1983). Criteria were applied repeatedly until no items could be excluded. Finally, the reliability indicators and the factorial structure adjustment indicators provided by the software were analyzed (Mislevy \& Bock, 1990). With the factorial structure of the instrument defined, were performed on SPSS correlation and regression analysis between the final scores of each factor and all demographic variables obtained.

\section{RESULTS}

\section{Factorial Analysis}

The adequacy of the correlation matrix was evaluated accordingly to the Kaiser criterion, which obtained a satisfactory value $(\mathrm{KMO}=0.926)$. Considering the parallel analysis results (Timmerman \& Lorenzo-Seva, 2011) and using the pre-established item maintenance/exclusion criteria (Gorsuch, 1983; Tabachnick \& Fidell, 2007), the final solution presented here grouped the questionnaire items into four factors.

The four observed factors obtained adequate reliability indices (>0.7) (Mislevy, \& Bock, 1990) and together explained $47.54 \%$ of the variance of the construct. The adjustment indexes indicate the adequacy of the proposed structure $(\mathrm{RMSEA}=0.048 ; \mathrm{CFI}=0.91 ; \mathrm{GFI}=0.98$; AGFI $=0.98 ; \mathrm{RSMR}=0.0431 ; \chi 2=1073.037 ; \mathrm{df}=662$ ).

Thus, the RMS proposed here included the following factors, which are presented in Table 2: (a) General retirement expectation: expectation of positive or negative experiences in retirement, feelings towards retirement, meaning pursuit, accomplishment, workers' rights, reward, desire to retire, adaptation to retirement; (b) Mental health and self-care: focus on the subject's well-being, mental health issues, anxiety, insecurity, aging; (c) Regulation and self-efficacy: personal organization, time management and financial management skills, autonomy in managing one's own life, interpersonal relationships management, recognition of goals and motivations, engaging in new activities, developing other life projects; and (d) Social insertion and recognition: changes in interpersonal relationships, social support, interpersonal relationships, changes in social position, social recognition, perceived usefulness to society, ability to perform socially relevant tasks.

\section{Correlations and Regressions}

Each factor generated a mean evaluation, and then correlation (Table 3 ) and regression analysis were performed for each of the factors and demographic variables - numeric variables were subjected to multiple linear regressions while categorical variables were submitted to logistic regressions. Factor 1, related to the general expectation of retirement, did not present significant correlations or regression values with any of the variables investigated. 


\begin{tabular}{|c|c|c|c|c|c|c|}
\hline & & & & Mean & SD & $\begin{array}{c}\text { Factor } \\
\text { Loading }\end{array}$ \\
\hline \multirow{12}{*}{$\begin{array}{l}\text { Factor 1: General } \\
\text { Retirement } \\
\text { Expectation } \\
(\alpha=0.905)\end{array}$} & $\begin{array}{l}\text { A aposentadoria é uma recompensa } \\
\text { por anos de trabalho }\end{array}$ & $\begin{array}{l}\text { A aposentadoria é uma punição } \\
\text { depois de trabalhar muitos anos }\end{array}$ & Item 11 & 5.45 & 1.09 & -0.665 \\
\hline & $\begin{array}{l}\text { A vida não tem sentido na } \\
\text { aposentadoria }\end{array}$ & $\begin{array}{r}\text { A vida tem sentido na } \\
\text { aposentadoria }\end{array}$ & Item35 & 5.39 & 1.04 & -0.605 \\
\hline & É fácil se adaptar à aposentadoria & $\begin{array}{r}\text { A aposentadoria é uma fase de } \\
\text { difícil adaptação }\end{array}$ & Item33 & 5.30 & 1.16 & -0.474 \\
\hline & $\begin{array}{l}\text { A aposentadoria é uma conquista } \\
\text { do trabalhador }\end{array}$ & $\begin{array}{r}\text { A aposentadoria é um prejuízo para } \\
\text { a sociedade }\end{array}$ & Item 24 & 5.04 & 1.12 & -0.446 \\
\hline & $\begin{array}{l}\text { A vida só tem sentido quando as } \\
\text { pessoas estão na ativa. }\end{array}$ & $\begin{array}{r}\text { A vida tem sentido mesmo após a } \\
\text { aposentadoria }\end{array}$ & Item8 & 5.04 & 1.25 & -0.516 \\
\hline & $\begin{array}{l}\text { A vida é menos prazerosa na } \\
\text { aposentadoria }\end{array}$ & $\begin{array}{r}\text { A vida na aposentadoria é mais } \\
\text { prazerosa }\end{array}$ & Item38 & 4.96 & 1.11 & -0.483 \\
\hline & $\begin{array}{l}\text { Os aposentados têm mais liberdade } \\
\text { para administrar suas vidas }\end{array}$ & $\begin{array}{l}\text { Os aposentados não têm liberdade } \\
\text { para administrar suas vidas }\end{array}$ & Item 48 & 2.18 & 1.07 & 0.690 \\
\hline & $\begin{array}{l}\text { Quando eu me aposentar, serei } \\
\text { dono do meu tempo }\end{array}$ & $\begin{array}{r}\text { Quando aposentar não terei } \\
\text { autonomia para gerenciar o meu } \\
\text { tempo }\end{array}$ & Item 36 & 2.12 & 1.16 & 0.782 \\
\hline & $\begin{array}{l}\text { A aposentadoria vai me trazer } \\
\text { felicidade }\end{array}$ & $\begin{array}{r}\text { A aposentadoria vai me trazer } \\
\text { tristeza }\end{array}$ & Item 37 & 2.10 & 1.27 & 0.716 \\
\hline & $\begin{array}{l}\text { Eu desejo que a minha } \\
\text { aposentadoria chegue logo }\end{array}$ & $\begin{array}{r}\text { Eu não quero me aposentar tão } \\
\text { cedo }\end{array}$ & Item 12 & 1.97 & 1.29 & 0.657 \\
\hline & $\begin{array}{l}\text { A aposentadoria é a garantia de } \\
\text { renda após anos de trabalho }\end{array}$ & $\begin{array}{r}\text { A aposentadoria não é qualquer } \\
\text { garantia de renda para o } \\
\text { trabalhador }\end{array}$ & Item 19 & 1.94 & 1.04 & 0.671 \\
\hline & $\begin{array}{l}\text { Quando penso em aposentadoria, } \\
\text { tenho a sensação de liberdade }\end{array}$ & $\begin{array}{l}\text { Aposentar não quer dizer que terei } \\
\text { liberdade para fazer o que quero }\end{array}$ & Item3 & 1.59 & 1.05 & 0.512 \\
\hline \multirow{7}{*}{$\begin{array}{l}\text { Factor 2: Mental } \\
\text { Health and Self- } \\
\text { care }(\alpha=0.807)\end{array}$} & $\begin{array}{l}\text { Com a aposentadoria as pessoas } \\
\text { têm mais tempo para cuidar de si }\end{array}$ & $\begin{array}{l}\text { A aposentadoria faz com que as } \\
\text { pessoas fiquem desleixadas }\end{array}$ & Item 1 & 4.35 & 1.26 & 0.531 \\
\hline & $\begin{array}{l}\text { Eu acredito que aposentadoria e } \\
\text { velhice caminham juntas }\end{array}$ & $\begin{array}{r}\text { Eu acredito que aposentadoria e } \\
\text { velhice são coisas distintas }\end{array}$ & Item 25 & 3.18 & 1.22 & -0.514 \\
\hline & $\begin{array}{l}\text { A aposentadoria é uma fase de } \\
\text { mais alegrias }\end{array}$ & $\begin{array}{r}\text { A aposentadoria é uma fase de } \\
\text { mais tristezas }\end{array}$ & Item6 & 3.12 & 1.76 & 0.347 \\
\hline & Os aposentados são desocupados & $\begin{array}{r}\text { Os aposentados se ocupam com } \\
\text { diversas atividades }\end{array}$ & Item 21 & 3.07 & 1.68 & 0.482 \\
\hline & $\begin{array}{l}\text { A aposentadoria não é uma } \\
\text { garantia do trabalhador }\end{array}$ & $\begin{array}{r}\text { A aposentadoria é um direito certo } \\
\text { do trabalhador }\end{array}$ & Item17 & 2.66 & 1.43 & 0.365 \\
\hline & $\begin{array}{l}\text { As pessoas esperam ansiosamente } \\
\text { o momento da aposentadoria }\end{array}$ & $\begin{array}{r}\text { As pessoas temem o momento da } \\
\text { aposentadoria }\end{array}$ & Item 16 & 2.58 & 1.40 & 0.663 \\
\hline & $\begin{array}{l}\text { Quando as pessoas se aposentam, } \\
\text { elas encontram novos objetivos } \\
\text { de vida }\end{array}$ & $\begin{array}{r}\mathrm{Na} \text { aposentadoria, os objetivos da } \\
\text { vida são perdidos }\end{array}$ & Item 15 & 2.00 & 1.45 & 0.588 \\
\hline \multirow{6}{*}{$\begin{array}{l}\text { Factor 3: } \\
\text { Regulation and } \\
\text { Self-efficacy } \\
(\alpha=0.879)\end{array}$} & $\begin{array}{l}\mathrm{Na} \text { aposentadoria as pessoas têm } \\
\text { dificuldade para organizar suas } \\
\text { vidas }\end{array}$ & $\begin{array}{r}\text { Os aposentados conseguem se } \\
\text { organizar melhor para fazer o que } \\
\text { desejam }\end{array}$ & Item34 & 4.80 & 1.25 & 0.651 \\
\hline & $\begin{array}{l}\text { Eu tenho medo de perder o contato } \\
\text { com meus amigos quando me } \\
\text { aposentar }\end{array}$ & $\begin{array}{r}\text { Vou poder investir mais nas } \\
\text { minhas amizades quando eu me } \\
\text { aposentar }\end{array}$ & Item 42 & 4.68 & 1.07 & 0.448 \\
\hline & $\begin{array}{l}\text { Os aposentados aproveitam menos } \\
\text { os prazeres da vida }\end{array}$ & $\begin{array}{r}\text { Os aposentados aproveitam mais } \\
\text { a vida }\end{array}$ & Item41 & 4.47 & 1.39 & 0.473 \\
\hline & $\begin{array}{l}\text { Na aposentadoria, as pessoas não } \\
\text { trabalham }\end{array}$ & $\begin{array}{r}\mathrm{Na} \text { aposentadoria, as pessoas } \\
\text { trabalham em algo novo }\end{array}$ & Item39 & 4.41 & 1.53 & 0.710 \\
\hline & $\begin{array}{l}\text { Pretendo desenvolver novos } \\
\text { projetos quando eu me aposentar }\end{array}$ & $\begin{array}{l}\text { Não pretendo desenvolver novos } \\
\text { projetos quando eu me aposentar }\end{array}$ & Item31 & 3.73 & 1.45 & 0.462 \\
\hline & $\begin{array}{l}\text { O contato com outras pessoas } \\
\text { diminui na aposentadoria }\end{array}$ & $\begin{array}{l}\mathrm{Na} \text { aposentadoria, é mais fácil } \\
\text { manter contato com outras pessoas }\end{array}$ & Item 23 & 3.65 & 1.57 & 0.749 \\
\hline
\end{tabular}




\begin{tabular}{|c|c|c|c|c|c|c|}
\hline & & & & Mean & SD & $\begin{array}{c}\text { Factor } \\
\text { Loading }\end{array}$ \\
\hline \multirow{4}{*}{$\begin{array}{l}\text { Factor 3: } \\
\text { Regulation and } \\
\text { Self-efficacy } \\
(\alpha=0.879)\end{array}$} & Eu tenho medo de me aposentar & $\begin{array}{r}\text { Eu não tenho medo de me } \\
\text { aposentar }\end{array}$ & Item 18 & 3.56 & 1.38 & 0.414 \\
\hline & $\begin{array}{l}\text { Depois da aposentadoria, as } \\
\text { pessoas podem fazer o que bem } \\
\text { entendem }\end{array}$ & $\begin{array}{r}\text { Depois da aposentadoria, as } \\
\text { pessoas não podem fazer o que } \\
\text { bem entendem }\end{array}$ & Item 43 & 2.76 & 1.41 & -0.526 \\
\hline & $\begin{array}{l}\text { Os aposentados podem organizar } \\
\text { sua rotina como quiserem }\end{array}$ & $\begin{array}{r}\text { Os aposentados não podem } \\
\text { organizar sua rotina como } \\
\text { quiserem }\end{array}$ & Item 28 & 2.60 & 1.44 & -0.591 \\
\hline & $\begin{array}{l}\text { Os aposentados já cumpriram sua } \\
\text { missão no mundo do trabalho }\end{array}$ & $\begin{array}{l}\text { Os aposentados não cumpriram sua } \\
\text { missão no mundo do trabalho }\end{array}$ & Item 46 & 2.30 & 1.07 & -0.449 \\
\hline \multirow{12}{*}{$\begin{array}{l}\text { Factor 4: Social } \\
\text { Insertion and } \\
\text { Recognition } \\
(\alpha=0.89)\end{array}$} & $\begin{array}{l}\text { Ainda serei útil quando me } \\
\text { aposentar }\end{array}$ & $\begin{array}{r}\text { Não me sentirei útil quando me } \\
\text { aposentar }\end{array}$ & Item9 & 5.34 & 1.07 & -0.536 \\
\hline & $\begin{array}{l}\text { A aposentadoria vai me } \\
\text { proporcionar qualidade de vida }\end{array}$ & $\begin{array}{l}\text { A aposentadoria não vai me } \\
\text { proporcionar qualidade de vida }\end{array}$ & Item 26 & 4.92 & 1.08 & -0.510 \\
\hline & $\begin{array}{l}\text { Aposentadoria é sinônimo de dever } \\
\text { cumprido }\end{array}$ & $\begin{array}{r}\text { Aposentadoria não é sinônimo de } \\
\text { dever cumprido }\end{array}$ & Item30 & 4.75 & 1.33 & -0.434 \\
\hline & $\begin{array}{l}\text { O aposentado não é útil a } \\
\text { sociedade }\end{array}$ & $\begin{array}{r}\text { O aposentado contribui para a } \\
\text { construção da sociedade }\end{array}$ & Item5 & 2.83 & 1.77 & 0.333 \\
\hline & $\begin{array}{l}\text { A aposentadoria vem acompanhada } \\
\text { de problemas de saúde }\end{array}$ & $\begin{array}{r}\text { Aposentadoria não vem } \\
\text { acompanhada de problemas de } \\
\text { saúde }\end{array}$ & Item7 & 2.73 & 1.68 & 0.517 \\
\hline & $\begin{array}{l}\text { Quando me aposentar, acho que } \\
\text { vai ser difícil me relacionar com } \\
\text { meus atuais amigos }\end{array}$ & $\begin{array}{r}\text { Quando eu me aposentar, vou } \\
\text { conseguir manter os amigos que } \\
\text { tenho hoje }\end{array}$ & Item 40 & 2.70 & 1.45 & 0.527 \\
\hline & $\begin{array}{l}\text { A aposentadoria traz problemas } \\
\text { psicológicos }\end{array}$ & $\begin{array}{l}\text { A aposentadoria não traz } \\
\text { problemas psicológicos }\end{array}$ & Item 45 & 2.56 & 1.22 & 0.606 \\
\hline & $\begin{array}{l}\mathrm{Na} \text { aposentadoria, a pessoa se } \\
\text { torna improdutiva }\end{array}$ & $\mathrm{O}$ aposentado é produtivo & Item 27 & 2.25 & 1.39 & 0.629 \\
\hline & $\begin{array}{l}\text { A aposentadoria é uma fase de } \\
\text { solidão }\end{array}$ & $\begin{array}{r}\text { A aposentadoria é a fase de } \\
\text { conviver com os amigos e família }\end{array}$ & Item29 & 2.06 & 1.15 & 0.629 \\
\hline & $\begin{array}{l}\text { A aposentadoria não é uma fase de } \\
\text { descanso }\end{array}$ & $\begin{array}{r}\text { A aposentadoria é uma fase de } \\
\text { descanso }\end{array}$ & Item 44 & 2.04 & 1.03 & 0.690 \\
\hline & $\begin{array}{l}\mathrm{O} \text { aposentado é um peso para a } \\
\text { sociedade }\end{array}$ & $\begin{array}{r}\mathrm{O} \text { aposentado contribui para a } \\
\text { sociedade }\end{array}$ & Item32 & 2.03 & 1.29 & 0.611 \\
\hline & $\begin{array}{l}\text { Os aposentados não são amparados } \\
\text { pela sociedade }\end{array}$ & $\begin{array}{r}\text { A sociedade dá suporte a seus } \\
\text { aposentados }\end{array}$ & Item2 & 1.82 & 1.08 & 0.552 \\
\hline
\end{tabular}

Factor 2 (Mental Health and Self-Care) had significant correlations with age $(\mathrm{r}=0.173 ; p<0.01)$, working years $(\mathrm{r}=-0.149 ; p<0.02)$ and proximity to retirement $(\mathrm{r}=0.237$; $p<0.001)$ and obtained for those variables an $\mathrm{R}^{2}=0.057$ $(p<0.01)$.

Factor 3 (Regulation and Self-Efficacy) had significant correlations with the variables gender $(\mathrm{rho}=-0.148$; $p<0.02)$ and proximity to retirement $(\mathrm{r}=-0.232 ; p<0.001)$. These same variables had low influence on the factor, represented by a Cox-Snell $\mathrm{R}^{2}=0.022$ concerning gender (Wald=17.66; $\mathrm{p}<0.001$ ) and an $\mathrm{R}^{2}=0.054$ concerning proximity to the event. A significant difference $(0.14$; $p<0.05)$ was found between the means of men $(\mathrm{M}=3.62$;
$\mathrm{SD}=0.49)$ and women $(\mathrm{M}=3.76 ; \mathrm{SD}=0.46)$ in this factor evaluation.

Finally, Factor 4 (Social Insertion and Recognition) obtained significant correlations with the variables gender $(\mathrm{rho}=0.193)$, race $(\mathrm{rho}=0.219)$ and proximity to retirement $(\mathrm{r}=0.26)$. An ordinal regression of those three variables obtained a Cox-Snell $\mathrm{R}^{2}=0.157$ (Wald $=18.49 ; p<0.001$ ), explaining $15.7 \%$ of the factor. A significant difference $(0.19$; $p<0.05)$ was found between the means of men $(\mathrm{M}=3.11$; $\mathrm{SD}=0.49)$ and women $(\mathrm{M}=2.91 ; \mathrm{SD}=0.49)$ in this factor evaluation. Also, results suggest that Black people tend to rate the factor more positively $(0.13 ; p<0.05)$. The joint analysis of the four factors resulted in a mean score of $3.32(\mathrm{SD}=0.24)$. 
Correlations

\begin{tabular}{|c|c|c|c|c|c|}
\hline & & $\begin{array}{c}\text { General Retirement } \\
\text { Expectation }\end{array}$ & $\begin{array}{c}\text { Mental Health and } \\
\text { Self-Care }\end{array}$ & $\begin{array}{c}\text { Regulation and Self- } \\
\text { Efficacy }\end{array}$ & $\begin{array}{l}\text { Social Insertion and } \\
\text { Recognition }\end{array}$ \\
\hline \multirow{3}{*}{ Age } & Pearson Correlation & -.039 & $-.173^{* *}$ & .072 & -.041 \\
\hline & Sig. (2 tailed) & .515 & .004 & .233 & .496 \\
\hline & $\mathrm{N}$ & 275 & 275 & 275 & 275 \\
\hline \multirow{3}{*}{ Working years } & Pearson Correlation & -.036 & $-.149^{*}$ & .028 & -.036 \\
\hline & Sig. (2 tailed) & .547 & .014 & .648 & .558 \\
\hline & $\mathrm{N}$ & 275 & 275 & 275 & 275 \\
\hline \multirow{3}{*}{$\begin{array}{l}\text { Proximity to } \\
\text { retirement }\end{array}$} & Pearson Correlation & -.083 & $.237^{* *}$ & $-.232^{* *}$ & $.260^{* *}$ \\
\hline & Sig. (2 tailed) & .182 & .000 & .000 & .000 \\
\hline & $\mathrm{N}$ & 262 & 262 & 262 & 262 \\
\hline \multirow{3}{*}{ Gender } & Correlation coefficient & .074 & .013 & $-.148^{*}$ & $.193^{* *}$ \\
\hline & Sig. (2 tailed) & .222 & .832 & .014 & .001 \\
\hline & $\mathrm{N}$ & 275 & 275 & 275 & 275 \\
\hline \multirow{3}{*}{ Race } & Correlation coefficient & -.001 & .113 & -.058 & $.219^{* *}$ \\
\hline & Sig. (2 tailed) & .984 & .062 & .338 & .000 \\
\hline & $\mathrm{N}$ & 275 & 275 & 275 & 275 \\
\hline
\end{tabular}

*. Correlation is significant at the 0.05 level (2 tailed).

**. Correlation is significant at the 0.01 level ( 2 tailed).

\section{DISCUSSION}

The investigation of retirement meanings involves approaching and measuring complex feelings and expectations of the individuals. In this study, the initial assumption was that there would be two possibilities of meaning for retirement (Cavalcante \& Minayo, 2012; Leandro-França et al., 2014) - the positive meanings and the negative meanings. In the scale proposed here, the six points presented to the respondents represent how positive or negative is the meaning he/she attributes to the phenomenon. Therefore, it is anchored that ratings up to 3.5 reflect more negative meanings and ratings over 3.5 should reflect more positive meanings.

Initially, the theoretical proposition suggested six factors to compose the meaning of retirement for Brazilian workers. However, the factorial analysis performed revealed only four components of the general meaning of retirement. The restructuring of the factors does not entail any prejudice to the scale, and it provides a better alignment between these results to previous retirement studies (e.g., Fisher et al., 2016; França et al., 2013, Leandro-França, 2016; Macêdo et al., 2017).

Factor 1 (General Retirement Expectation) reflects the common social beliefs about retirement. This part of meaning is characteristic of the pre-retirement phase in which respondents to this survey find themselves: when workers often report expectations of how their retirement should be (Macêdo et al., 2017; Magalhães et al., 2004). From a practical point of view, this factor should have a greater impact on retirement adjustment patterns - especially considering that it alone explains $31.37 \%$ of the meaning attributed to the phenomenon - as workers' anticipations directly reflect their adaptation to retirement (Magalhães et al., 2004).

The average evaluation of the first factor $(M=3.59$; $\mathrm{SD}=0.07$ ) suggests that workers' expectations relate to positive and negative experiences in balanced proportions, with a slight tendency towards positive expectations. When observing the averages per item, the results corroborate the findings of previous studies: retirement is seen as a reward for the worker (Cavalcante \& Minayo, 2012; Oliveira et al., 2009; Shibata 2006), but brings with it the anguish linked to autonomy and the ability to manage life without working (Zanelli et al., 2010).

The second factor (Mental Health and Self-Care) expresses a more affective content of the meaning of retirement, generally composed of feelings of anxiety related to the need to develop new projects (Cavalcante \& Minayo, 2012; Duarte \& Melo-Silva, 2009; Zanelli et al., 2010), and expectations about affective experiences in general (Cavalcante \& Minayo, 2012; Shibata, 2006). Moreover, this factor arises from the association pointed by Debert (2013) between retirement and aging. The overall evaluation of the factor is negative $(\mathrm{M}=2.99 ; \mathrm{SD}=0.58)$, and only Item 1 was positively evaluated $(\mathrm{M}=4.35 ; \mathrm{SD}=1.27)$, while all others had a mean evaluation of 3.18 or lower. These data reveal the workers' fear of retirement and the sense of life goals losses (Cavalcante \& Minayo, 2012; Macêdo et al., 2017; Shibata, 2006; Zanelli et al., 2010). 
In addition, the correlations found point to the difference between two groups already described by Magalhães et al. (2004): workers in the remote phase of retirement and workers in the approximate phase of retirement. It is noticed that the older the respondent $(\mathrm{r}=-0.173)$, the more working years he/she has $(\mathrm{r}=-0.149)$ and the less time left until retirement $(\mathrm{r}=0.237)$, the more negatively the Factor 2 is evaluated. In other words, workers in the remote phase of retirement tend to develop a more positive perspective on mental health and self-care in retirement, while workers in the approximate phase tend to develop the opposite perspective. This happens because, in the approximate phase, the foreshadowing of no longer having work activities or social situations permeated by it highlights for the changes in the social roles the respondent plays and, especially, the change in the way he/ she is perceived others (Magalhães et al., 2004).

The third factor (Regulation and Self-Efficacy) refers to the ability of retired workers to manage their own lives, and it was positively assessed by respondents $(\mathrm{M}=3.7 ; \mathrm{SD}=0.48)$. The items with more extreme evaluations point to the belief that retirees can better organize their lives to invest in what they want, but that does not mean that they are free to do only what pleases them. This perception is strongly associated with the idea that retirement is a stage of life in which people are free to invest in projects that, during the working life, were neglected (Cavalcante \& Minayo, 2012, Duarte \& Melo-Silva, 2009; França \& Carneiro, 2009; Leandro-França et al., 2014; Macêdo et al., 2017).

Factor 4 (Social Insertion and Recognition), unlike other factors, reflects an interpersonal component of retirement, which recognized as an important factor for retirement adjustment (França, 2009; França, \& Carneiro, 2009); This factor deals mainly with interpersonal relationships and the post-retirement social role. The average factor rating is negative $(\mathrm{M}=3 ; \mathrm{SD}=0.5)$ and it reveals some internal contradictions: respondents indicate that they will still find themselves useful after retirement (Item 9: $\mathrm{M}=5.34$; $\mathrm{SD}=1.07$ ), but also indicate that the retiree is a burden to society (Item 32 : $\mathrm{M}=2.03 ; \mathrm{SD}=1.29$ ). The ambivalence of responses in this factor summarizes aspects already discussed in the literature. In general, workers realize that interrupting work activities points to an important change in social environment (Duarte \& Melo-Silva, 2009; França et al. 2013; Zanelli et al., 2010), with a negative impact on established relationships and social status in a way that the workers themselves see the retiree can be a hindrance to society. However, when the individual thinks about himself, the criterion seems to change, and he/she does not see a retired person as a social problem. This shift in perspective may be partly related to the need to maintain a positive self-concept (Baumeister, 2010): healthy human beings tend to avoid creating negative images of themselves as a form of self-preservation.
The differences in Factors 3 and 4 assessments between men and women reveal interesting information about the meanings attributed to retirement. According to the results, and contrary to what was previously observed in the literature (Debert, 2013; Dias, 2017; Fernandes \& Garcia, 2010), women evaluate the Social Insertion and Recognition factor in retirement more negatively than men; the opposite is true for evaluations of Regulation and Self-Efficacy factor. Those results might indicate that there are some differences regarding the relevance of work in the construction of women's identity (Barreto \& Heloani, 2018): working is an emancipating activity for a woman, and it is through it that she achieves social recognition. In that sense, getting a job is a way out of marginalization and towards independency. For men, on the other hand, disruption in work activities does not compromise his social status as a man, and moreover it gives him the autonomy to manage his life, so it is understandable that the averages between these groups oscillate in this way.

A small difference was identified in the construction of retirement meaning between Black people and White people: Black respondents provided higher averages for Social Insertion and Recognition. One of the theories in social psychology that might explain is the Theory of Relative Deprivation (Stouffer et al., 1949) - it is expected that White-dominated work environments might frustrate Black workers due to the comparison with the dominant group. Therefore, the insertion and recognition outside this context and away from the group comparison that it brings seems more promising to that minority group.

Finally, the data reveals a negative meaning attributed to the retirement phenomenon $(\mathrm{M}=3.32 ; \mathrm{SD}=0.25)$. The results obtained in this study once again point to the paradox presented in the phenomenon of retirement (Guerson et al., 2018; Leandro-França et al., 2014; Leandro-França, 2016; Macêdo et al., 2017; Oliveira, et al., 2009): there is an overall positive expectation about retirement (Factor 1: $\mathrm{M}=3.59 ; \mathrm{SD}=0.27$ ) and an idea of freedom associated to it (Factor 3: $\mathrm{M}=3.7 ; \mathrm{SD}=0.48$ ). On the other hand, workers report experiences of anguish and insecurity (Factor 2: $\mathrm{M}=2.99$; $\mathrm{SD}=0.76$ ) and recognize the negative impact of changing social roles in retirement (Factor 4: $\mathrm{M}=3 ; \mathrm{SD}=0.5$ ). Assessments of Factors 2 and 4 may also foreshadow retirement anxiety - a concern about the unknown (Duarte \& Melo-Silva, 2009) and the stigma (Fernandes et al., 2016) of retirement, marked by a negative perception of the phenomenon (Fletcher \& Hansson, 1991).

The negative attributions found resume one of the initial discussions of the present study: the construction and strengthening of negative retirement meanings and perceptions in the working population today can result in a population marked by poor retirement adjustment and experiences. 


\section{FINAL CONSIDERATIONS}

This study sought to construct and search for validity evidences of a retirement meaning scale for active workers and to investigate the meanings attributed to retirement by this population. A Retirement Meaning Scale was developed as a tool to assist in designing more accurate interventions for pre-retirement workers. The statistical analysis conducted show how the social groups differ in this meaning attribution, since some specific variables, such as proximity to retirement (Magalhães et al., 2004), and gender (Barreto \& Heloani, 2018; Debert, 2013; Dias, 2017; Fernandes \& Garcia, 2010) effectively impact the results.

Among the limitations of the study, the choice to collect data virtually, as well as the use of a semantic differential scale, limits the research to a richer and highly educated sample. To access lower social strata, it may be necessary to adapt the scale, and invest in a paper and pen questionnaire with face-to-face application.

Finally, it is necessary to recognize that the time period in which these data were collected (May/2017 - October/2017) was a politically and socially troubled period in Brazil. Even though this provides an important historical record of the phenomenon studied, it makes data very volatile, since this is a very specific picture of this social context. Therefore, it is expected that this research will unfold into others that complement or contrast the information exposed so far.

\section{REFERENCES}

Antunes, M. H., Soares, D. H. P., \& Moré, C. L. O. O. (2015). Repercussões da aposentadoria na dinâmica relacional familiar na perspectiva do casal. Psico, 46(4), 432-441. https://doi. org/10.15448/1980-8623.2015.4.19495

Bardin L. (1977). Análise de conteúdo. Edições 70.

Barreto, M. M. S., \& Heloani, R. (2018). Gênero e trabalho. In R. Mendes (Ed.), Dicionário de saúde e segurança do trabalhador: Conceitos, definições, história e cultura (559560). Proteção.

Baumeister, R. F. (2010). The self. In R. F. Baumeister \& E. J. Finkel (Eds.), Advanced social psychology (pp. 139-175). Oxford University Press.

Bendassolli, P. F., \& Gondim, S. M. G. (2014). Significados, sentidos e função psicológica do trabalho: Discutindo essa tríade conceitual e seus desafios metodológicos. Avances en Psicología Latinoamericana, 32(1), 131-147. https://doi. org/10.12804/ap132.1.2014.09

Boehs, S. D. T. M., Medina, P. F., Bardagi, M. P., Luna, I. N., \& Silva, N. (2017). Revisão da literatura latino-americana sobre aposentadoria e trabalho: perspectivas psicológicas. Revista Psicologia Organizações e Trabalho, 17(1), 54-61. https://doi. org/10.17652/rpot/2017.1.11598

Bressan, M. A. L. C., Mafra, S. C. T., França, L. H. F. P., Melo, M. S. S., \& Loreto, M. D. S. (2012). Trabalho versus aposentadoria: desvendando sentidos e significados. Oikos: Família e Sociedade em Debate, 23(1), 226-250. https://www.oikos.ufv. br/index.php/oikos/article/view/76

Cavalcante, F. G., \& Minayo, M. C. S. (2012). Autópsias psicológicas e psicossociais de idosos que morreram por suicídio no Brasil. Ciência \& Saúde Coletiva, 17(8), 19431954. https://doi.org/10.1590/S1413-81232012000800002

Debert, G. G. (2013). Feminismo e velhice. Sinais Sociais, 8(22), 1538. http://www.sesc.com.br/wps/wcm/connect/aa3fe0d5-61a443e7-9e7d-b0c74bffa2a3/Revista+-\%20+Sinais Sociais 22 web.pdf?MOD=AJPERES\&CACHEID=aa3fe 0 $\overline{\mathrm{d} 5}-61 \mathrm{a} 4-\overline{43} \mathrm{e} 7-$ 9e7d-b0c74bffa2a3.

Denton, F. T., \& Spencer, B. G. (2009). What is retirement? A review and assessment of alternative concepts and measures. Canadian Journal on Aging, 28(1), 63-76. https://doi.org/10.1017/ S0714980809090047

Duarte, C. V., \& Melo-Silva, L. L. (2009). Expectativas diante da aposentadoria: Um estudo de acompanhamento em momento de transição. Revista Brasileira de Orientação Profissional,
10(1), 45-54. http://pepsic.bvsalud.org/pdf/rbop/v10n1/ v10n1a07.pdf.

Felix, Y. T. M., \& Catão, M. F. (2013). Envelhecimento e aposentadoria por policiais rodoviários. Psicologia \& Sociedade, 25(2), 420-429. http://www.scielo.br/pdf/psoc/ v25n2/19.pdf.

Fernandes, M. D. G. M., \& Garcia, L. G. (2010). O sentido da velhice para homens e mulheres idosos. Saúde e Sociedade, 19(4), 771-783. http://www.scielo.br/pdf/sausoc/v19n4/05.pdf.

Fernandes, P. C. M., Marra, A. V., \& Lara, S. M. (2016). Metamorfoses identitárias na pré-aposentadoria de servidores públicos. Revista de Carreiras e Pessoas (ReCaPe), 6(1), 8699. https://doi.org/10.20503/recape.v6i1.28025

Fisher, G. G., Chaffee, D. S., \& Sonnega, A. (2016). Retirement timing: A review and recommendations for future research. Work, Aging and Retirement, 2(2), 230-261. https://doi. org/10.1093/workar/waw001

Fletcher, W. L., \& Hansson, R. O. (1991). Assessing the social components of retirement anxiety. Psychology and Aging, 6(1), 76-85. https://doi.org/10.1037/0882-7974.6.1.76

Fouad, N. A., \& Bynner, J. (2008). Work transitions. American Psychologist, 63(4), 241-251. https://doi.org/10.1037/0003066X.63.4.241

França, L. H. F. P. (2008). O desafio da aposentadoria: O exemplo dos executivos do Brasil e da Nova Zelândia. Rocco.

França, L. H. F. P. (2009). Influências sociais nas atitudes dos 'top' executivos em face da aposentadoria: Um estudo transcultural. Revista de Administração Contemporânea, 13(1), 17-35. https://doi.org/10.1590/S1415-65552009000100003

França, L. H. F. P., Amorim, S. M., Souza, A. P., \& Schuabb, T. C. (2017). Autobiografia orientada para avaliar vida, carreira e planejar para a aposentadoria. Revista Brasileira de Orientação Profissional, 18(2), 249-258. https://doi.org/10.26707/1984$7270 / 2017 \mathrm{v} 18 \mathrm{n} 2 \mathrm{p} 249$

França, L. H. F. P., \& Carneiro, V. L. (2009). Programas de preparação para a aposentadoria: Um estudo com trabalhadores mais velhos em Resende (RJ). Revista Brasileira de Geriatria e Gerontologia, 12(3), 429-448. https://doi.org/10.1590/18099823.2009.00010

França, L. H. F. P., Menezes, G. S., Bendassolli, P. F., \& Macedo, L. S. S. (2013). Aposentar-se ou continuar trabalhando? O que influencia essa decisão? Psicologia: Ciência e Profissão, 33(3), 548-563. https://doi.org/10.1590/S1414-98932013000300004 
França, C. L., Murta, S. G., Negreiros, J. L., Pedralho, M., \& Carvalhedo, R. (2013). Intervenção breve na preparação para aposentadoria. Revista Brasileira de Orientação Profissional, 14(1), 99-110. http://pepsic.bvsalud.org/pdf/rbop/v14n1/10. pdf.

Gorsuch, R. (1983). Factor analysis (2nd ed.). Lawrence Erlbaum Associates.

Guerson, L. R. S. C., França, L. H. F. P., \& Amorim, S. M. (2018). Life Satisfaction in retirees who are still working. Paidéia, 28, e2812. https://doi.org/10.1590/1982-4327e2812

IBM Corp. (2012). IBM SPSS Statistics for Windows, Version 21.0. IBM Corp.

Leandro-França, C. (2016). Efeito de programas de preparação para aposentadoria: um estudo experimental (Tese de Doutorado). http://repositorio.unb.br/handle/10482/21219.

Leandro-França, C., \& Murta, S. G. (2014). Fatores de risco e de proteção na adaptação à aposentadoria. Psicologia Argumento, 32(76), 33-43. https://doi.org/10.7213/psicol.argum.32.076 DS03

Leandro-França, C., Murta, S. G., \& Iglesias, F. (2014). Planejamento da aposentadoria: Uma escala de mudança de comportamento. Revista Brasileira de Orientação Profissional, 15(1), 75-84. https://doi.org/10.1037/t65886-000

Lorenzo-Seva, U., \& Ferrando, P.J. (2013). FACTOR 9.2 A comprehensive program for fitting exploratory and semiconfirmatory factor analysis and IRT Models. Applied Psychological Measurement, 37(6), 497-498. https://doi. org/10.1177/0146621613487794

Lorenzo-Seva, U., Timmerman, M. E., \& Kiers, H. A. L. (2011). The Hull method for selecting the number of common factors. Multivariate Behavioral Research, 46, 340-364. https://doi.or $\mathrm{g} / 10.1080 / 00273171.2011 .564527$

Macêdo, L. S. S., Bendassolli, P. F., \& de Torres, T. L. (2017). Representações sociais da aposentadoria e intenção de continuar trabalhando. Psicologia \& Sociedade, 29, e145010. https://doi.org/10.1590/1807-0310/2017v29145010

Machado, C. N. C., \& Lucas, M. G. (2017). Aposentadoria: Como professores vivenciam este momento?. Revista de Carreiras e Pessoas (ReCaPe), 7(2), 576-588. https://doi.org/10.20503/ recape.v7i2.32753

Magalhães, M. O., Krieger, D. V., Vivian, A. G., Straliotto, M. C. S., \& Poeta, M. P. (2004). Padrões de ajustamento na aposentadoria. Aletheia, 19, 57-68. http://pepsic.bvsalud.org/ pdf/aletheia/n19/n19a06.pdf.

Mislevy, R. J., \& Bock, R. D. (1990). BILOG 3 Item analysis and test scoring with binary logistic models. Scientific Software. https://doi.org/10.1177/01466216970214006

Oliveira, J.C. (2018). Aposentadoria e trabalho. In R. Mendes (Ed.), Dicionário de saúde e segurança do trabalhador: Conceitos, definições, história e cultura (pp. 139-140). Proteção.

Oliveira, C., Torres, A. R. R., \& Albuquerque, E. S. (2009). Análise do bem estar psicossocial de aposentados de Goiânia. Psicologia em Estudo, 14(4), 749-757. https://doi.org/10.1590/ S1413-73722009000400015

Osgood, C. E. (1952). The nature and measurement of meaning. Psychological Bulletin,49(3), 197-231. https://doi.org/10.1037/ $\mathrm{h} 0055737$
Osgood, C. E., Suci, G. J., \& Tannenbaum, P. H. (1957). The measurement of meaning. Board of Trustees of the University of Illinois.

Pasquali, L. (2010). O diferencial semântico. In. L. Pasquali (Ed.), Instrumentação psicológica: Fundamentos e práticas (pp. 262-272). Artmed.

Rafalski, J. C., Noone, J. H., O'Loughlin, K., \& Andrade, A. L. (2017). Assessing the process of retirement: A cross-cultural review of available measures. Journal of Cross-Cultural Gerontology, 32(2), 255-279. https://doi.org/10.1007/s10823017-9316-6

Richins, M. L. (1994). Valuing things: The public and private meanings of possessions. Journal of Consumer Research, 21, 504-521. https://doi.org/10.1086/209414

Rodrigues, A. T. N. A., Andrade, L. N., Silva, B. M. F., Lira, N. C. M. \& Antloga, C. S. X., (2014). Aposentadoria nas Ciências do Trabalho. In Caderno de Resumos do VI Congresso Brasileiro de Psicologia Organizacional e do Trabalho. Associação Brasileira de Psicologia Organizacional e do Trabalho.

Shultz, K. S., \& Wang, M. (2011). Psychological perspectives on the changing nature of retirement. American Psychologist, 66(3), 170-179. https://doi.org/10.1037/a0022411

Shibata, L. H. (2006). "Em busca de um novo caminho": O PósCarreira como oportunidade de realização de potencialidades (Dissertação de mestrado não-publicada). Pontifícia Universidade Católica de São Paulo, São Paulo, Brasil.

Stouffer, S. A., Suchman, E. A., DeVinney, L. C., Star, S. A., \& Williams Jr, R. M. (1949). The American soldier: Adjustment during army life. (Studies in social psychology in World War II, vol 1). Princeton University Press.

Tabachnick, B. G., \& Fidell, L.S. (2007). Using multivariate statistics. Pearson Education.

Timmerman, M. E., \& Lorenzo-Seva, U. (2011). Dimensionality assessment of ordered polytomous items with parallel analysis. Psychological Methods, 16, 209-220. https://doi.org/10.1037/ a0023353

Triandis, H. C. (2002). Subjective culture. Online Readings in Psychology and Culture, 2(2), 6. https://doi.org/10.9707/23070919.1021

Yamamoto, O. H. (2015). Trabalho. In P. F. Bendassolli \& J. E. Borges-Andrade (Eds.), Dicionário de psicologia do trabalho e das organizações (pp. 641-647). Casa do Psicólogo.

Yannoulas, S. C. (2018). Feminização (feminilização) do trabalho. In R. Mendes (Ed.), Dicionário de saúde e segurança do trabalhador: Conceitos, definições, história e cultura (pp. 559-560). Proteção.

Zanelli, J. C. (2015). Aposentadoria e pós-carreira. In P. F. Bendassolli \& J. E. Borges-Andrade (Eds.), Dicionário de psicologia do trabalho e das organizações (pp. 59-68). Casa do Psicólogo.

Zanelli, J. C. (2012). Processos psicossociais, bem-estar e estresse na aposentadoria. Revista Psicologia: Organizações e Trabalho, 12(3), 329-340. http://pepsic.bvsalud.org/pdf/rpot/v12n3/ v12n3a07.pdf.

Zanelli, J. C., Silva, N., \& Soares, D. H. P. (2010). Orientação para aposentadoria nas organizações de trabalho: construção de projetos para o pós-carreira. Artmed. 